\title{
Positive Thinking Training Increases Tolerance Levels of Ambiguity and Distress in Men with Hemophilia
}

\author{
Masoumeh Beiranvand ${ }^{1}$, Majid Keramati Moghadam², Reza Sabounchi ${ }^{3}$, Ghasem Asadpour ${ }^{2}$, Nahid Jahani ${ }^{1}$ and \\ Azam Ghafuori ${ }^{4}$ \\ 1. Department of Psychology, Faculty of Social Sciences, Imam Khomeini International University, Qazvin, Iran \\ 2. Department of Sport Management, Faculty of Physical Education and Sport Sciences, Islamic Azad University Borujerd Branch, \\ Borujerd, Iran \\ 3. Faculty of Physical Education and Sport Sciences, Islamic Azad University of Borujerd, Borujerd, Iran \\ 4. Department of Educational Psychology, Faculty of Psychology and Education, Allameh Tabataba'i University, Tehran, Iran
}

\begin{abstract}
Background: Low levels of ambiguity tolerance and distress tolerance observed in people with hemophilia lead to decreasing their function and quality of life. How positive thinking training program affects tolerance of ambiguity and distress is not completely understood. Accordingly, the purpose of this study was to explain the role of positive thinking training in men with hemophilia of Lorestan province and examine its effectiveness on ambiguity tolerance and distress tolerance. Method: The statistical population of this study included 129 patients with hemophilia who were a member of Hemophilia Association of Lorestan province. Thirty (30) patients who were randomly selected were randomly assigned to the experimental group $(N=15)$ and the control group $(N=15)$. The design of present study was an experimental design with the pre- and post-test that the experimental group received a positive thinking training. Eight techniques that were adapted from Seligman's techniques were used to teach positive thinking. These techniques were held in 8-two hours sessions (two sessions per week). The instrument of this study included Simons and Gaher's distress tolerance scale and McLean and David's ambiguity tolerance questionnaire. The collected data were analyzed by the analysis of covariance. Results: The results of this study showed that there was a significant difference between scores of post-test and follow-up of two variables in two groups. Conclusion: The amount of ambiguity tolerance and distress tolerance increased in the experimental group in comparison with control group.
\end{abstract}

Key words: Positive thinking, ambiguity tolerance, distress tolerance, hemophilia.

\section{Introduction}

Hemophilia is one of the rare bleeding disorders associated with the inherited $\mathrm{x}$ gene appearing in men in which blood was not clot normally resulted by the lack of sufficient blood-clotting proteins [1-3]. There are two types of hemophilia including type A and B [2, 3]. However, hemophilia requires a continuous management and treatment, most patients with hemophilia will have a healthy lifestyle if they have a proper treatment and self-care [3]. Many people with hemophilia suffer from chronic pain due to arthritis, as

Corresponding author: Majid Keramati Moghadam, Ph.D Candidate, Department of Sport Management. Faculty of Physical Education and Sport Sciences, Islamic Azad University Borujerd Branch, Borujerd, Iran. well as acute pain of bleeding in joints. The age and illness severity index typically describes a small part of the life-related health variance in the hemophilia. Therefore, more variance can be studied by factors such as the pain coping and its acceptance. Pain coping in a hemophiliac population usually involves targeted efforts to manage pain or reduce its effect, regardless of the success or failure of these efforts. Furthermore, pain coping sometimes includes negative thoughts about pain, fear, anger, and catastrophic pain that probably reflect emotional distress associated with pain rather than efforts at coping. In addition, pain coping is sometimes characterized as efforts to overcome pain by the control, reduction, and avoidance of pain. On the other hand, pain acceptance includes a tendency to 
experience pain without its reduction, prevention, and change. Among the studies that compared the effect of these three factors (pain coping, negative thoughts, and pain acceptance) in chronic pain conditions, some studies showed that the factors related to the pain acceptance more than other factors were associated with an improved performance. The pain acceptance is affected by the functional state, while pain coping is under the influence of emotional distress. Negative thoughts are mediator for the effects of pain severity on depression and interference with daily life in patients with sickle cell disease and low acceptance is due to the effects of catastrophizing in chronic pain on physical and mental function. Moreover, negative thoughts affect the mental quality of life, because these thoughts reduce the pain acceptance. It has shown that negative thoughts increased the effects of pain severity on the mental quality of life in hemophilic population. This result emerges a very important role of negative thoughts in the mental quality of life. Interventions to improve mental adjustment in hemophilia patients can focus on the reduction of negative thoughts about pain [4]. Chronic physical illnesses (such as asthma, diabetes, cardiovascular disease, hemophilia, etc.) are conditions that cannot be cured at the moment, but can be managed with a proper treatment [5]. We have currently shown that positive thinking training increased significantly self-efficacy and emotion regulation in hemophiliac men [6]. These outcomes showed the important role of positive thinking training that may work as a fundamental strategy to tackle with stressful situations of life. On the other hand, it showed that optimistic and positive attitudes toward failures improve mental health and our thoughts can also increasingly affect our emotional problems [7]. In general, positive thinking and the avoidance of negative thoughts are good strategies for dealing more effectively with problems [6,8]. Furthermore, emotional distress tolerance is the ability to experience and tolerate negative psychological states [9]. There are evidences that show the change in perceptions of disease and treatment has a beneficial effect on mental distress and quality of life. Psychological distress is largely defined as a state of emotional suffering characterized by symptoms of depression and anxiety that it can be considered as a normal emotional response to a stressor but can meet the diagnostic criteria for a psychiatric disorder with other symptoms. The low emotional distress tolerance can be associated with a range of mental health disorders such as post-traumatic stress disorder and depression [9]. Distress tolerance can be defined as the ability to prevent the response to the behavioral option that it can reduce distress instantly in stressful states [10]. It is expected that psychological deficiencies (for example, tolerance of suffering and pain catastrophizing) are associated with health high-risk behaviors. Distress tolerance is defined as real disability or its perception to tolerate negative emotional states [11]. Moreover, distress tolerance skills may be effective in the reduction of the number of target behaviors, especially high-risk behaviors and dangerous suicides [12]. In addition, the lack of distress tolerance is a risk factor for many forms of psychiatry. It has shown that skills training such as anxiety and distress tolerance may be an effective potential intervention to deal with veterans suicidal behaviors to response their unique needs [13]. Since hemophiliac patients have a low ambiguity tolerance and distress tolerance due to their illness problems, resulted by decreasing their function and quality of life. Accordingly, we attempted to investigate these two variables in these patients by the training of positive thinking style. Hence, the purpose of this study was to explain the role of positive thinking training on ambiguity tolerance and distress tolerance in patients with hemophil.

\section{Methodology}

\subsection{Study Design}

This study was an applied and semi-experimental research with pre-, post-test, and follow-up design, using control group. 


\subsection{Subjects}

The statistical population of this study was all men who were a member of Hemophilia Association of Lorestan Province $(N=129)$. Thirty (30) patients with hemophilia were randomly selected from this population. They were randomly assigned to the experimental group $(N=15)$ and the control group $(N$ $=15$ ). Written informed consent was obtained by all participants

\subsection{Instruments and Tasks}

The instrument of this study included Simons and Gaher's distress tolerance scale, McLean and David's ambiguity tolerance questionnaire, and the positive thinking training, including eight techniques that have been adapted from the techniques used by Seligman, Patterson, and other psychologists. The distress tolerance scale is a self-measurement index of emotional distress tolerance that is designed by Simons and Gaher [14]. The items of this scale measures distress tolerance on the basis of individual's ability for emotional distress tolerance, mental distress assessment, the rate of attention to negative emotions in case of occurrence, and regulatory actions for the distress relief. This scale has 15 items and 4 subscales, including emotional distress tolerance, absorption by negative emotions, mental disturbance estimation, and regulation efforts to relieve distress. The items of this scale were scored on the basis of five-point Likert's scale. High scores on this scale indicate a high distress tolerance. The alpha coefficients for these subscales were respectively $0.72,0.82,0.78,0.70$, and 0.82 for the whole scale. The intra-class correlation coefficient was 0.61 after six months. It has also been shown that this scale has a good initial criterion and convergent validity [15]. McLean's ambiguity tolerance questionnaire has 22 items [16]. The subject answers to any questions on a 5-point Likert's scale from completely agree to completely disagree. This questionnaire is designed on the basis of revised definitions of ambiguity characteristics and individual responses to their perceptions. The validity and reliability of this questionnaire have been reported above 0.60 [17]. The positive thinking training includes eight techniques that have been adapted from the techniques used by Seligman, Patterson, and other psychologists. These eight techniques are: 1-introduction and direction (the explanation of how to train and determine work routines), 2-the identification of personal capabilities and use them, 3 - the recall of three positive events/the mention of blessings, 4-Personal heritage, 5-a thank letter and meeting, 6-the active and constructive response, 7-work with patience (Avoid rush), and 8-the stabilization of happiness. These techniques were held in eight sessions of 2 hours (two sessions a week).

\subsection{Procedure}

The positive thinking training was applied as an independent and interventional variable in the experimental group and ambiguity tolerance and distress tolerance were measured as dependent variables before and after the use of independent variable. The independent variable was presented to the experimental group in eight-2 hour sessions. The follow-up stage was conducted on the control and experimental groups for one month after the end of training. The results of both groups were compared before and after the presentation of the training.

\subsection{Statically Analysis}

The analysis of covariance was used to assess the effect of the pre-test variable (positive thinking teaching) on the dependent variable (post-test scores of ambiguity tolerance and emotional distress tolerance) due to the nature of study. Also, the assumption of homogeneity of regression slopes and variances was surveyed before the covariance analysis. Levene's test was used to survey the assumption of homogeneity of variances. Furthermore, the Kolmogorov-Smirnov test was used to determine the normal distribution of scores in two variables. SPSS statistical software (version 21) 
was used to statistically analyze all data. Its significance level was set at $p<0.05$. All data were presented as mean \pm SD as well.

\section{Results}

The results in Table 1 show subjects' number, the mean, standard deviation, minimum, and maximum scores of research variables in the separation of groups. The results in Table 2 show the mean and standard deviation in the pre- and post-test of the control and experimental groups in two variables of ambiguity tolerance and emotional distress tolerance by the separation of groups.

The analysis of covariance to assess the effect of the pre-test variable (positive thinking teaching) on the dependent variable (post-test scores of ambiguity tolerance and emotional distress tolerance) is presented in Table 3. In addition, assumptions of covariance analysis, including the homogeneity of regression slopes and homogeneity of variances, and the assumption of the normal distribution of scores were evaluated before the covariance analysis (Tables 3-5).

Table 1 The frequency distribution of demographic characteristics in the sample.

\begin{tabular}{llll}
\hline Age & Experimental group & Control group & Total \\
\hline Number & 15 & 15 & 30 \\
Mean & 31.67 & 30.07 & 30.87 \\
SD & 6.10 & 7.96 & 7.02 \\
Minimum & 18 & 18 & 18 \\
Maximum & 41 & 45 & 45 \\
\hline
\end{tabular}

Table 2 Descriptive indicators of variables.

\begin{tabular}{|c|c|c|c|c|c|}
\hline variable & & Groups & Number & Mean & SD \\
\hline \multirow{6}{*}{ Ambiguity tolerance } & \multirow{2}{*}{ Pre-test } & Experimental & 15 & 63.80 & 8.62 \\
\hline & & Control & 15 & 63.07 & 6.84 \\
\hline & \multirow{2}{*}{ Post-test } & Experimental & 15 & 70.13 & 9.67 \\
\hline & & Control & 15 & 61.87 & 7.43 \\
\hline & \multirow{2}{*}{ Follow up } & Experimental & 15 & 66.73 & 9 \\
\hline & & Control & 15 & 61.27 & 7.11 \\
\hline \multirow{6}{*}{ Emotional distress tolerance } & \multirow{2}{*}{ Pre-test } & Experimental & 15 & 51.40 & 10.41 \\
\hline & & Control & 15 & 42.40 & 5.23 \\
\hline & \multirow{2}{*}{ Post-test } & Experimental & 15 & 55.93 & 9.55 \\
\hline & & Control & 15 & 41.47 & 7.23 \\
\hline & \multirow{2}{*}{ Follow up } & Experimental & 15 & 54.13 & 9.16 \\
\hline & & Control & 15 & 41.13 & 7.03 \\
\hline
\end{tabular}

Table 3 The assumption of homogeneity of regression slopes.

\begin{tabular}{lllllll}
\hline Variance resources & & Sum of squares & df & Mean square & $F$ & Sig \\
\hline \multirow{2}{*}{ Ambiguity tolerance } & Post-test & 14.48 & 1 & 14.48 & 1.18 & 0.28 \\
& Follow up & 2.87 & 1 & 2.87 & 0.33 & 0.56 \\
\hline \multirow{2}{*}{ Emotional distress tolerance } & Post-test & 6.80 & 1 & 6.80 & 0.39 & 0.53 \\
& Follow up & 25.10 & 1 & 25.10 & 2.22 & 0.14 \\
\hline
\end{tabular}

Table 4 The results of assumption of equality of variances.

\begin{tabular}{llllll}
\hline Variables & & $F$ & $\mathrm{df}_{1}$ & $\mathrm{df}_{2}$ & Sig. \\
\hline \multirow{2}{*}{ Ambiguity tolerance } & Post-test & 3.43 & 1 & 28 & 0.07 \\
& Follow up & 1.19 & 1 & 28 & 0.28 \\
\hline \multirow{2}{*}{ Emotional distress tolerance } & Post-test & 3.50 & 1 & 28 & 0.07 \\
& Follow up & 2.31 & 1 & 28 & 0.14 \\
\hline
\end{tabular}


Table 5 The survey of assumption of normal distribution of scores.

\begin{tabular}{llll}
\hline Variables & & The amount of Kolmogorov-Smirnov statistic & Sig. \\
\hline \multirow{2}{*}{ Ambiguity tolerance } & Post-test & 3.43 & 0.93 \\
& Follow up & 1.19 & 0.61 \\
\hline \multirow{2}{*}{ Emotional distress tolerance } & Post-test & 3.50 & 0.48 \\
& Follow up & 2.31 & 0.27 \\
\hline
\end{tabular}

Table 6 The results of covariance analysis of life expectancy and coping skills.

\begin{tabular}{lllllllll}
\hline Variables & $\begin{array}{l}\text { Dependent } \\
\text { variable }\end{array}$ & $\begin{array}{l}\text { Change } \\
\text { resources }\end{array}$ & $\begin{array}{l}\text { Sum of } \\
\text { squares }\end{array}$ & $\mathrm{df}$ & Mean squares & $F$ & Sig. & Effect size \\
\hline \multirow{3}{*}{$\begin{array}{l}\text { Ambiguity } \\
\text { tolerance }\end{array}$} & \multirow{2}{*}{ Post-test } & Pre-test & $1,750.17$ & 1 & $1,750.17$ & 141.77 & 0.01 & 0.84 \\
& \multirow{2}{*}{ Follow up } & Group & 423.29 & 1 & 423.29 & 34.29 & 0.01 & 0.55 \\
& & Pre-test & 16.17 & 1 & 16.17 & 192.87 & 0.01 & 0.87 \\
Emotional & \multirow{2}{*}{ Post-test } & Group & 168.84 & 1 & 168.84 & 20.13 & 0.01 & 0.42 \\
distress & & Group & 226.46 & 1 & 226.46 & 13.59 & 0.01 & 0.33 \\
tolerance & \multirow{2}{*}{ Follow up } & Pre-test & $1,551.36$ & 1 & $1,551.36$ & 131.68 & 0.01 & 0.83 \\
& & Group & 134.80 & 1 & 134.80 & 11.44 & 0.01 & 0.29 \\
\hline
\end{tabular}

The results in Table 3 showed that there was no equivalence between the group and the pre-test scores in the post-test and follow-up stages. Therefore we can state that regression slopes are homogeneous (the variable of ambiguity tolerance in the post-test stage: $p_{\text {value }}=0.28, F=1.18$, in the follow up stage: $p_{\text {value }}=$ $0.56, F=0.33$; the variable of emotional distress tolerance in the post-test stage: $p_{\text {value }}=0.53, F=0.39$, in the follow up stage: $p_{\text {value }}=0.14, F=2.22$ ).

The results in Table 4 showed that there was no significant difference between variances of two groups in the post-test and follow up stages (the variable of ambiguity tolerance in the post-test stage: $p_{\text {value }}=0.07$, $F=3.43$, in the follow up stage: $p_{\text {value }}=0.28, F=1.19$; the variable of emotional distress tolerance in the post-test stage: $p_{\text {value }}=0.07, F=3.50$, in the follow up stage: $p_{\text {value }}=0.14, F=2.31$ ).

The results in Table 5 showed that the distribution of the scores was close to the normal distribution and there was no significant difference.

The auxiliary random variable (pre-test) had a significant relationship with dependent variables (post-test and follow-up variables) represented in Table 6. Also, there was a significant effect of factor between subjects of group in the variable of ambiguity tolerance (post-test: $F=34.29$ and follow up: $F=20.13$ ) and the variable of emotional distress tolerance (post-test: $F=$ 13.59 and follow up: $F=11.44$ ) after the adjustment of the pre-test scores. Therefore, the results showed a significant difference between scores of the post-test and the follow up of both variables in the two groups. It showed that the amount of ambiguity tolerance and emotional distress tolerance has increased in the experimental group after the positive thinking training.

\section{Discussion}

The results of the present study showed that positive thinking training increased the level of ambiguity tolerance and distress tolerance in men with hemophilia. The analysis of the scores by covariance test showed that the effect of the pre-test variable (positive thinking training) on the dependent variable (post-test scores of ambiguity tolerance and emotional distress tolerance) was significant. These results are acknowledged by previous studies [5, 6, ,8,18, 19]. Our positive thinking training resulted in changing the thinking style in these patients and affected two psychological variables such as ambiguity tolerance and emotional distress tolerance. The concept of ambiguity tolerance is one of mechanisms that have attracted an increasing attention in recent years. Ambiguity tolerance is defined as an individual's 
tendency to show a negative reaction to events and vague situations, independent of its probability of occurrence or consequences [20]. In a situation that demands evaluation or choice, the perceived presence of ambiguity is threatening and presents a cognitive challenge in the form of desired but absent or inaccessible information. Consequently, ambiguity could be a barrier that hampers decision making and prediction. Intolerance of ambiguity is the aversion to this lack of information, whereas ambiguity tolerance is the degree of acceptance of, or even attraction to, this lack of information [21]. In addition, the power of ambiguity tolerance is an important feature that plays a key role in the initiation and continuation of risk-taking that leads to success. The rate of anxiety that is produced in response to an uncertain collection of received information received about a situation is ambiguity tolerance and it is an individual's specific and fixed characteristic. The severity of the reaction to the received ambiguity reflects individuals' ambiguity tolerance [22]. Emotional distress tolerance is a discussed another variable by the researcher in this study. The findings of Rizou, et al. [23] showed that perceptions predict mental distress disease and the quality of life in patients with epilepsy. They also showed that psychological distress is predicted by time schedule, personal control, therapeutic control, and emotional representations. Since the necessity of positive thinking and positivism has been proven in previous studies, so that they have shown the effectiveness of this thinking style on the improvement of quality of life and its relationship with mental health. These findings were confirmed in the present study. Hence, teaching positive thinking training to men with hemophilia may count as an effective tool to increase the tolerance levels of ambiguity and emotional distress. Trick et al. [5] have shown that a degree of perseverative negative thinking and depression, anxiety, and emotional distress may seem logical in people with long-term conditions. Accordingly, perseverative negative thinking (such as worry and rumination) predicts depression and worse medical outcomes. Based on the perspective of behavioral science and psychology, the mental state, the physical state, and the experience of pain or happiness have the origin in rumination and style of our thoughts. Fortunately, psychologists have achieved a strategy to change the pessimism and negative thought. Positive ideation can be used to effectively counter worry, so that it has shown that the repeat of training in the replacement of worry with different forms of positive ideation can cope with unpleasant and unintentional properties of worry [18]. Positive thinking had a positive relationship with health and could play a positive role in health. The positive thinking training for hemophilia patients is very valuable and useful to improve their positive relationship with themselves and others, so that they are able to have a good feel about themselves and control emotions and feelings. It has shown that people with low tolerance have greater exaggeration in pain [12]. As a result, the topic of positive thinking has high importance due to the importance and value of positive thinking on the health and mental relaxation in the society, especially the results of this study can be used in the future in counseling centers and mental health clinics.

\section{Conclusion}

The present study aimed to examine the effectiveness of positive thinking training on ambiguity tolerance and distress tolerance in men with hemophilia and to discuss about implication of positive thinking in clinical and psychological setting. In order to develop psychological training programs, it is necessary to measure their effectiveness on psychological characters and quality of life of hemophiliac population. As physical activity and exercise training programs as a powerful lever have a positive effect on the prevention and treatment of physiological diseases in healthy and unhealthy subjects [24-29], psychological training such as positive thinking training can be also used as an 
essential plan, effective tool and important lever to improve and promote psychological factors including the tolerance levels of ambiguity and distress in congenital disease population such as hemophiliac patients. Therapists can persuade patients and help them to understand and improve their strengths using the strategies of positive psychology.

\section{Acknowledgment}

We thank all men with hemophilia that participated in this project.

\section{Conflict of Interest}

The authors declare no conflict of interest.

\section{References}

[1] Dolan G, Benson G, Duffy A, Hermans C, Jimenez-Yuste $\mathrm{V}$, Lambert T, et al. Haemophilia B: Where are we now and what does the future hold? Blood reviews. 2018; 32(1):52-60.

[2] Schrader J, White M, Silberstein PT. Hemophilia. xPharm: The Comprehensive Pharmacology Reference: Elsevier Inc.; 2011. p. 1-4.

[3] Schrader J, White M, Silberstein P, Shiozawa Y. Hemophili: Reference Module in Biomedical Research; 2015.

[4] Elander J, Robinson G, Mitchell K, Morris J. An assessment of the relative influence of pain coping, negative thoughts about pain, and pain acceptance on health-related quality of life among people with hemophilia. PAIN ${ }^{\circledR}$. 2009; 145(1-2):169-75.

[5] Trick L, Watkins E, Windeatt S, Dickens C. The association of perseverative negative thinking with depression, anxiety and emotional distress in people with long term conditions: A systematic review. Journal of psychosomatic research. 2016; 91:89-101.

[6] Beiranvand M, Moghadam MK, Sabounchi R, Delphan M, Ghafuori A, Moghadam KR. The effectiveness of positive thinking training on self-efficacy and emotion regulation in men with hemophilia. Trends Med. 2019; 19(4):1-5.

[7] zinivand $m$, kazemi $f$, salimi h. education for parents of children with cancer 7-3 benchmark hospital in Tehran in 1392. Counseling Culture and Psycotherapy. 2014; 5(19):34-50.

[8] Shafiei K. The effectiveness of positive thinking training in a group method on the reduction of test anxiety and the control of anger in high school students in schools of
Jiroft city. Iran: Islamic Azad University Marvdasht Branch; 2009.

[9] Holliday SB, Pedersen ER, Leventhal AM. Depression, posttraumatic stress, and alcohol misuse in young adult veterans: The transdiagnostic role of distress tolerance. Drug and alcohol dependence. 2016; 161:348-55.

[10] Seo J-W. How do you restrain yourself from avoidance when distressed? Distress tolerance and affective associations of avoidance/withstanding behaviors in college-aged heavy drinkers. Personality and Individual Differences. 2017; 111:1-5.

[11] Banducci AN, Connolly KM, Vujanovic AA, Alvarez J, Bonn-Miller MO. The impact of changes in distress tolerance on PTSD symptom severity post-treatment among veterans in residential trauma treatment. Journal of anxiety disorders. 2017; 47:99-105.

[12] Emami AS, Woodcock A, Swanson HE, Kapphahn T, Pulvers K. Distress tolerance is linked to unhealthy eating through pain catastrophizing. Appetite. 2016; 107:454-9.

[13] Denckla CA, Bailey R, Jackson C, Tatarakis J, Chen CK. A novel adaptation of distress tolerance skills training among military veterans: Outcomes in suicide-related events. Cognitive and Behavioral Practice. 2015; 22(4):450-7.

[14] Simons JS, Gaher RM. The Distress Tolerance Scale: Development and validation of a self-report measure. Motivation and Emotion. 2005; 29(2):83-102.

[15] Esmaeilinasab M, Andami Khoshk A, Azarmi H, Samar Rakhi A. The predicting role of difficulties in emotion regulation and distress tolerance in students' addiction potential. Research on addiction. 2014; 8(29):49-63.

[16] McLain DL. The MSTAT-I: A new measure of an individual's tolerance for ambiguity. Educational and psychological measurement. 1993; 53(1):183-9.

[17] Agajani S, Narimani M, Ariapooran S. Comparing of Perfectionism and Tolerance of Ambiguity in Gifted and Non- gifted Students. Journal of Exceptional Children. 2011; 11(1):83-90.

[18] Eagleson C, Hayes S, Mathews A, Perman G, Hirsch CR. The power of positive thinking: Pathological worry is reduced by thought replacement in Generalized Anxiety Disorder. Behaviour research and therapy. 2016; 78:13-8.

[19] Kakavand A, Shams Esfandabad H, Beiranvand M. Effect of the positive thinking education to the mothers on their children externalizing disorders reduction. Journal of School Psychology. 2016; 4(4):125-42.

[20] Salmani B, Hasani J. The role of intolerance of uncertainty and positive metacognitive beliefs in obsessive compulsive disorder and depression. Razi Journal of Medical Sciences. 2016; 23(142):64-72.

[21] Arquero JL, Polvillo CF, Hassall T, Joyce J. Relationships between communication apprehension, 
ambiguity tolerance and learning styles in accounting students. Revista de Contabilidad-Spanish Accounting Review. 2017; 20(1):13-24.

[22] McLain DL, Kefallonitis E, Armani K. Ambiguity tolerance in organizations: definitional clarification and perspectives on future research. Frontiers in psychology. 2015; 6:344.

[23] Rizou I, De Gucht V, Papavasiliou A, Maes S. Illness perceptions determine psychological distress and quality of life in youngsters with epilepsy. Epilepsy \& Behavior. 2015; 46:144-50.

[24] Khoramshahi S, Kordi MR, Delfan M, Gaeini A, Safa M. Effect of Five Weeks of High-Intensity Interval Training on the Expression of miR-23a and Atrogin-1 in Gastrocnemius Muscles of Diabetic Male Rats. Iranian Journal of Endocrinology and Metabolism. 2017; 18(5):361-7.

[25] Ghareghani P, Shanaki M, Ahmadi S, Khoshdel AR, Rezvan N, Meshkani R, et al. Aerobic endurance training improves nonalcoholic fatty liver disease (NAFLD) features via miR-33 dependent autophagy induction in high fat diet fed mice. Obesity research \& clinical practice. 2018; 12(1):80-9.

[26] Delphan M, Rashidlamir A, Delphan F. Resting plasma AgRP levels response to exercise-conjugated diet and only diet. Biology of Sport. 2012; 29(1):45-50.

[27] Delphan M, Rashidlamir A, Delfan F, Izadpanah N, Rahbarizade F. The effects of two weight loss protocol on resting plasma concentration of IL-6 in overweight and obese health sedentary female of college students. 22nd International Congress on Pediatrics; 2010.

[28] Delphan M, Agha Alinejad H, Delfan M, Dehghan S. Intratumoral Effects of Continuous Endurance Training and High Intensity Interval Training on Genes Expression of miR-21 and bcl-2 in Breast Cancer Bearing Female mice. Iranian Quarterly Journal of Breast Diseases. 2017; 10(2):49-57.

[29] Delphan M, Rashidlamir A, Ebrahimi-Atri A, Saadatnia A. The effect of two weight loss protocols on plasma concentration of agouti related peptide (AgRP) in elite wrestlers. Jundishapur Scientific Medical Journal. 2013; 12(3):229-41. 